\title{
Synthesis and operation of an FFT-decoupled fixed-order RFP plasma control system based on identification data
}

\author{
K Erik J Olofsson ${ }^{1}$, Per R Brunsell ${ }^{1}$, Emmanuel Witrant ${ }^{2}$, \\ James R Drake ${ }^{1}$ \\ ${ }^{1}$ School of Electrical Engineering, Royal Institute of Technology (KTH \\ Stockholm), Sweden, Association EURATOM-VR \\ ${ }^{2}$ Control Systems Department, UJF/GIPSA-Lab, Grenoble University, France \\ E-mail: erik.olofsson@ee.kth.se
}

\begin{abstract}
Recent developments and applications of system identification methods for the reversed-field pinch (RFP) machine EXTRAP T2R have yielded plasma response parameters for decoupled dynamics. These datasets are the fundament for a real-time implementable fast fourier transform (FFT) decoupled discrete-time fixed-order strongly-stabilizing synthesis as described in this work. Robustness is assessed over the dataset by bootstrap calculation of the sensitivity transfer function worst-case $\mathcal{H}_{\infty}$-gain distribution. Output-tracking and magnetohydrodynamic (MHD) mode $m=1$-tracking is considered in the same framework simply as two distinct weighted traces of a performance channel output-covariance matrix as derived from the closed-loop discrete-time Lyapunov equation. The behaviour of the resulting multivariable controller is investigated with dedicated T2R experiments.
\end{abstract}

PACS numbers: 52.55.Tn, 52.35.Py, 52.55.-s

Submitted to: Plasma Phys. Control. Fusion 


\section{Introduction}

Stabilization of the reversed-field pinch (RFP) plasma by magnetic feedback systems is a proven concept in theory and in practice. Experimental control of multiple resistive-wall modes (RWMs) have been shown feasible [1,2]. Recently, a refurbished implementation [3] of the original intelligent-shell (IS) [4] furthermore showed successful sustainment of nonzero nonaxisymmetric radial magnetic field boundary conditions throughout plasma discharges. The work described here primarily strives to design and deploy the fourier-mode decoupled controller, a reconfiguration enabled by harnessing results from novel experiments that measure the external plasma response in the RFP $[5,6]$. Although fourier mode-controllers (MCs) have been tested before, these are quite far away from the set of controllers synthesised in this work. First, previous RFP MCs have not been (rigorously) tuned based on plasma-response identification data and second, controller synthesis methods have been spartan [7], at best, but mostly absent.

A central assumption adhered to when designing MCs is the concept of mode rigidity. Mode rigidity means that the MHD mode structure remains intact during instability (RWM) onset and controller intervention. The hypothesis of mode rigidity is central to the formulation of modal circuit equations required for MCs, e.g. [8]. Recent experiments [9] indicate that the concept is not, for tokamaks at least, entirely robust. In this work we assume that modal circuit equations are a sound startingpoint. This is conventional for RFPs.

State-of-the-art magnetic control for the RFP is at the time of writing encapsulated in the acronym CMC or clean mode control, as introduced in [10]. We will parallel this design from a quite different (and in some sense generalised) language here.

Provisional studies of experimental behaviour is found in the last section, and we will comment on potential inadequacies of pure discrete fourier transform (DFT) type decoupling. Though FFT-decoupling is nice in theory, the real-world anisotropic vacuum vessel could severly challenge the attained performance in practice. The studies of 3D conducting structures' influence for tokamak RWM control [11] is related to this issue.

\subsection{EXTRAP T2R reversed-field pinch}

EXTRAP T2R RFP, housed in the Alfvén Laboratory, Stockholm, Sweden, is built upon the skeletal remainings of the OHTE experiment originally constructed at General Atomics in San Diego [12]. T2R is a relatively small experiment with major toroidal radius $R=124 \mathrm{~cm}$, a circular cross-section, and a confined-plasma minor radius of $a=18 \mathrm{~cm}$. As a high-aspect ratio $R / a \approx 7 \mathrm{RFP}$ machine, the cylindrical 1D-magnetohydrodynamic (MHD) plasma approximation is believed to be a suitable approximation for macroscopic behaviour on the resistive-shell time-scale. An assortment of key parameters for T2R are listed in table 1. Plasma discharge lengths $\tau_{p l} \gtrsim 20 \mathrm{~ms}$ are only attained with magnetic feedback stabilization activated. This is a direct implication of the relatively short eddy-decay shell time-constant $\tau_{w}$. The experimental success of feedback RWM stabilization is loosely quantified by the ratio $\tau_{p l} / \tau_{w} \lesssim 10$.

The minor radii listed in table 1 can be size-sorted as $a<r_{s}<r_{w}<r_{c}$. This relates to figure 1: $r_{s}$ (blue), $r_{w}$ (grey), $r_{c}$ (red) and plasma $a$ (vaguely pink). The 
plasma-containing vacuum vessel is positioned just inside the sensor coils at $r_{s}$, and is not depicted in figure 1 . This means that the sensor coils are sandwiched between the vessel and the resistive shell (a.k.a. wall) at $r_{w}$ [12]. The vessel magnetic field diffusion time constant $\tau_{v}$ is about two orders of magnitude faster than the shell's $\tau_{w}$, thus $\tau_{v}$ is ignored for the basic dynamical expression (4) encountered below.

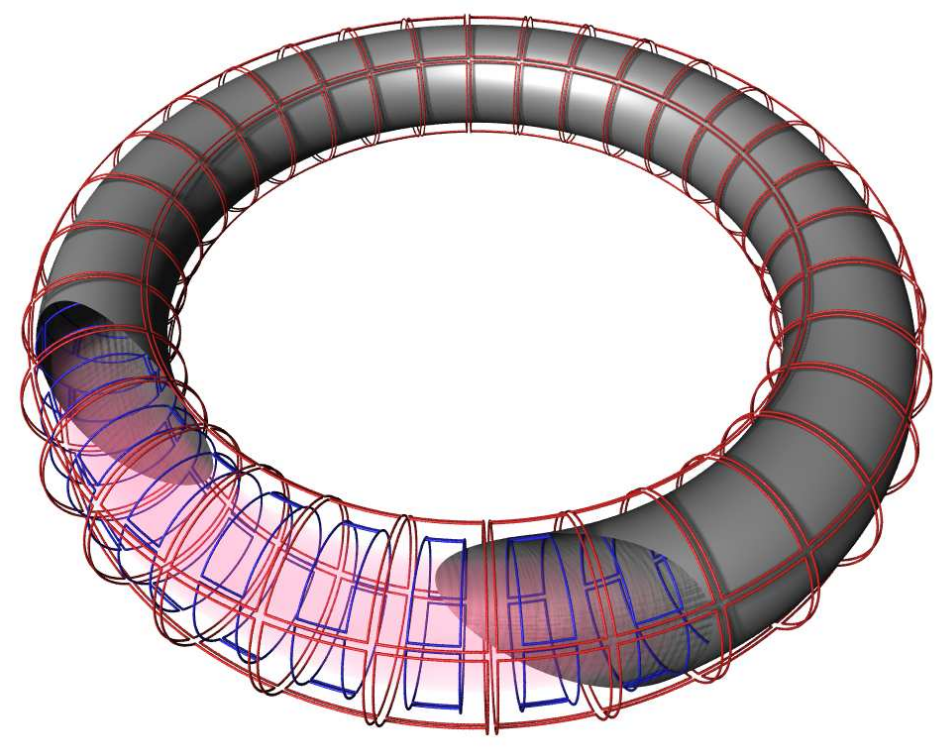

Figure 1. T2R feedback accoutrements: a dense arrangement of active coils (red, outermost) and sensor coils (blue, innermost).

Table 1. Machine parameters for T2R

\begin{tabular}{lll} 
Symbol & Value/order & Description/comment \\
\hline$I_{p l}$ & $\sim 100 \mathrm{kA}$ & plasma current \\
$\tau_{p l}$ & $10-100 \mathrm{~ms}$ & plasma shot duration \\
$\tau_{w}$ & $\sim 10 \mathrm{~ms}$ & resistive wall time \\
$\tau_{v}$ & $\sim 50 \mu \mathrm{s}$ & vessel wall time \\
$T_{s}$ & $100 \mu \mathrm{s}$ & digital sampling time, controller cycle \\
$a$ & $0.183 \mathrm{~m}$ & plasma minor radius \\
$R$ & $1.24 \mathrm{~m}$ & plasma major radius \\
$r_{w}$ & $0.198 \mathrm{~m}$ & resistive-shell radial position \\
$r_{s}$ & $0.197 \mathrm{~m}$ & sensor coil radial position \\
$r_{c}$ & $0.238 \mathrm{~m}$ & actuator coil radial position
\end{tabular}

\subsection{Signal notations}

Consider figure 1 . We will denote by $\tilde{\mathbf{y}}(t)$ and $\tilde{\mathbf{u}}(t)$ vector signals of sorted stacked scalars $\tilde{y}_{i}$ and $\tilde{u}_{i}$ corresponding to sensor values and actuator amplifier input respectively. These signals are sorted in the sense that $\tilde{y}_{i}$ and $\tilde{u}_{i}$ indexes the same surface position on the torus. The vector dimension is 64 despite figure 1 depicts 
$4 \times 32=128$-sized arrays. The reason is that, currently, T2R sensors and actuator are economically connected by pairwise subtraction such that the outboard-inboard and the up-down saddle coil pairs share signals. The result is 2 signals for each of the 32 equidistant positions along the toroidal angle, describing horizontal and vertical magnetic field perturbations. A drawback is the loss of even- $m$ mode numbers due to the signal subtraction, but for T2R the set of $m=1$ mode-numbers is the most important. It is well known that the unstable portion of T2R RFP eigenmodes are $m=1$-modes with low $|n|[13,14]$. Also encountered in this work are the signals $\tilde{\mathbf{r}}(t)$, and $\tilde{\mathbf{w}}(t)$ respectively denoting reference and dither vectors.

The non-tilde signal vector $\mathbf{y}$ is the spatial DFT of its tilde companion and is obtained as follows.

$$
\begin{aligned}
& (\mathcal{T} \tilde{\mathbf{y}})_{i}(t)=\sum_{j=0}^{N-1} W_{j i}\left(\tilde{y}_{j, A}(t)+\iota \tilde{y}_{j, B}(t)\right) \\
& y_{j}(t)=\left\{\begin{array}{l}
\operatorname{Re}(\mathcal{T} \tilde{\mathbf{y}})_{j}(t), j=0 \ldots 31 \\
\operatorname{Im}(\mathcal{T} \tilde{\mathbf{y}})_{j-32}(t), j=32 \ldots 63
\end{array}\right.
\end{aligned}
$$

In (1), $\iota^{2}=-1, W_{j i}=e^{-2 \pi j i \iota / N}, N=32$ and pair-subscripts $(j, A),(j, B)$ respectively maps to the corresponding indexes for outboard-inboard $A$ and top-down $B$ signals at toroidal angle $j$. Scalars $y_{j}, j=0 \ldots 63$ then constitute the vector $\mathbf{y}$. Vectors $\mathbf{u}, \mathbf{r}, \mathbf{w}$ $\in \mathbb{R}^{64}$ are similarly defined by replacement of $y$ in (1). DFT vector index $j$ is related to MHD mode number $n$ via the mapping $n(j)=n_{j}, n_{j}$ being the $j$ th component of $\mathbf{n}^{T}=(0) \ldots 15,-16 \ldots 15,-16 \ldots-1$.

We will denote by $s$ the Laplace transform parameter [15] at any occurence of continuous-time transfer functions throughout the text. $z$ will be reserved for the $z$-transform $[15,16]$ parameter where applicable for discrete-time system descriptions. Recall that $z^{-1} x(k)=x(k-1)$, where $k$ is the sample index. A sample index increment for T2R represents a sample-interval of $T_{s}=100 \mu \mathrm{s}$, or equivalently a sample-frequency of $f_{s}=1 / T_{s}=10 \mathrm{kHz}$.

\subsection{Background and setting: the intelligent-shell and mode-control}

Feedback emulation of a perfectly conducting boundary was first proposed in reference [4], viz. the intelligent-shell (IS). The basic IS scheme utilizes an array of sensor and current-carrying actuator saddle-shaped coils, figure 1, partially or fully covering the surface of the toroidal resistive shell. Originally, the response of an actuator is only affected by the sensor centered at the same toroidal and poloidal coordinate. The actuator response is a magnetic field anti-directed relative to the radial magnetic field perturbation detected by the incident sensor saddle coil. Thus, a 'simulated' perfect-conductor surface eddy-current counteracting the perturbation is produced, and consequently magnetic field diffusion through the container is hindered.

At T2R the following IS feedback has been implemented [3].

$$
\tilde{\mathbf{u}}=M_{e q} F_{P I D}(s)(\tilde{\mathbf{r}}-\tilde{\mathbf{y}})
$$

Compared to the classic IS, (2) is slightly generalised since a reference signal $\tilde{\mathbf{r}}$ can be tracked for any sensor. In (2), $M_{e q}$ is a tridiagonal (approximately diagonal) matrix designed for two reasons: $i$ ) to detach (minimize) the influence of actuators to adjacent sensors, ideally only exciting the incident sensor and ii) to equalize the individual amplitudes of the power amplifiers driving the actuator coils [17]. Additionally, $F_{P I D}(s)$ is a diagonal proportional-integral-derivative (PID) [18] controller; tuned [3], symptomatically, without knowledge of the experimental plasma response. The study 
[19] also had this weakness: no available experimental values for plasma growth-rates, albeit the theoretical shape of the RFP spectrum was considered in this design.

In principle the counterworking actuator response could be improved by harnessing knowledge of the spatial structure of the perturbation. In linear RFP stability theory, independently growing helical fourier modes can be predicted, and these structures have been observed experimentally. A feedback system that identifies and reacts on predefined spatial patterns is generally denoted a mode-controller (MC). Two complications arise when actually designing this system. First, experimental parameters for the plasma dynamics are needed, and second, the experimental spatial eigenmode structure is required. Technically, detailed tuning of IS designs also suffers from this problem, however, omitting experimental plasma response identification can perhaps be more easily motivated for IS since the decentralized nature of the IS control system has no possibility of distinguishing between spatial perturbation patterns anyway. With any MC controller comes the pretention to taylor the feedback response to the modal dynamics which cannot be done unless experimental information on this dynamics is acquired.

The scope of this study is the design and deployment of a statically decoupled MC derived from experimental plasma parameters in this particular decoupling. Specifically, the eigenmode structure will be discrete fourier modes, implemented via the fast fourier transform (FFT) [20, 21]. The prescription of helical fourier modes is theoretically sound when the plasma obeys cylindrical symmetry equilibrium, and resistive-shell time-scale MHD contained in a cylindrical homogeneous thin shell. This last assumption is known to be false for T2R. There are insulating cuts in the shell to avoid electrical short-circuits. Nevertheless this type of spatial feedback has been applied extensively in the literature, although usually in a more explorative manner than will be presented below.

More specifically, we will, in section 2, design an FFT-decoupled control system of the form

$$
\tilde{\mathbf{u}}=M_{e q} W_{D F T}^{-1} F_{F O}(s)\left(L_{r} \mathbf{r}+W_{D F T} \tilde{\mathbf{y}}\right)
$$

where matrix $W_{D F T}$, implicitly defined by (1), betokens the linear FFT operation. $F_{F O}(s)$ is a diagonal fixed-order controller and $L_{r}$ is a diagonal constant matrix. The elements of $F_{F O}(s)$ and $L_{r}$ are developed in detail in section 2. Note that the reference $\mathbf{r}$ is defined in mode-space here, in contrast to $\tilde{\mathbf{r}}$ in (2).

\subsection{Measured fourier-mode parametric external plasma response}

The parametric external plasma response model prescribed for identification and control synthesis here is the conventional $[13,22,14]$ linear independent-mode dynamics

$$
\tau_{m, n} \dot{x}_{m, n}-\hat{\gamma}_{m, n} x_{m, n}=x_{m, n}^{e x t}, \forall m>0, n \in \mathbb{Z}
$$

where $x_{m, n}^{e x t}=x_{m, n}^{e r r}+x_{m, n}^{a p p}$ is a driving term dichotomized into an error part and a externally applied known part (active coils). The normalized modal growth-rate $\hat{\gamma}_{m, n}$ encapsulates the MHD content of (4) and the modal time-constant $\tau_{m, n}$ stems from electromagnetic diffusion through a passive resistive cylindrical shell. It must be clearly understood that what is prescribed in this work is only the model structure of (4). We do not trust any numerical values not originating from system identification experiments interpreted in this form (4). Specifically, we impose dynamics (4) for each fourier mode $(m, n)$ and this implies aliasing to DFT component $i$ which is 
approximated by truncation. A comparable truncation is invoked in [10]. It is neither purpose nor scope here to detail identification algorithms and procedures $[5,6,23]$ but we will need to provide enough information on model parameterization to introduce the matrices required in section 2 . It is sufficient to consider here the model

$$
G_{i}\left(z, \theta_{i}\right)=\sum_{(m, n) \in \mathcal{K}_{n(i)}} G_{m, n}\left(z, \theta_{i}\right)
$$

that takes the role of the plant $G$ in figure 3. The parameter vector $\theta_{i}$ is detailed below. System (5) is constituted by zero-order-hold discretizations [16] of (4)

$$
G_{m, n}(z, \theta)=\frac{\alpha \hat{c}_{m, n} \hat{b}_{m, n} \frac{1}{\hat{\gamma}_{m, n}}\left(\hat{d}_{m, n}-1\right) z^{-1}}{1-\hat{d}_{m, n} z^{-1}}
$$

using $\hat{d}_{m, n}=e^{\frac{\hat{\gamma}_{m, n} T_{s}}{\hat{\tau}_{m, n} \tau}}$. In (6) $\hat{c}_{m, n}, \hat{b}_{m, n}$ and $\hat{\tau}_{m, n}$ are constant geometric factors [5] associated with T2R's configuration. The truncated set of aliased mode-numbers summed over in (5) is

$$
\mathcal{K}_{n}=\left\{\begin{array}{lll}
(1, n), & (1, n-32), & (1, n+32), \\
(3, n), & (3, n-32), & (3, n+32), \\
(5, n), & (5, n-32), & (5, n+32)
\end{array}\right\}
$$

throughout this study. For each fourier component $i$, the imposed first-order discretetime model of the disturbance system $H$ in figure 3 is

$$
H_{i}\left(z, \theta_{i}\right)=\frac{b_{i} z^{-1}}{1-a_{i} z^{-1}}
$$

which is essentially modeling the specific part $x_{m, n}^{e r r}$ of $x_{m, n}^{e x t}$ in (4). For the $i$ th spatial fourier component the parameter vector

$$
\theta_{i}^{T}=\left(\begin{array}{ccccc}
\alpha_{i} & \tau_{i} & \hat{\gamma}_{m=1, n(i)} & a_{i} & b_{i}
\end{array}\right)
$$

has been estimated from experimental data. In addition to mode subset truncation (7) another crucially simplifying approximation has been made. As suggested from (9) only one plasma growth-rate parameter is determined; the one corresponding to mode $(m=1, n)$, i.e. the very first one in subset (7). All other growth-rates are defaulted to a constant value $\hat{\gamma}_{m, n}=-1$, basically the vacuum response to external perturbations $[5,6]$.

For each fourier component $i$ there is available

(i) a set of scalars $\left\{\alpha_{i}^{(j)}\right\}_{j=1 \ldots N_{\alpha}}$ with arithmetic mean $\left\langle\alpha_{i}\right\rangle$

(ii) a set of scalars $\left\{\tau_{i}^{(j)}\right\}_{j=1 \ldots N_{\tau}}$ with arithmetic mean $\left\langle\tau_{i}\right\rangle$

(iii) a set of scalars $\left\{\hat{\gamma}_{i}^{(j)}\right\}_{j=1 \ldots N_{\hat{\gamma}}}$ with arithmetic mean $\left\langle\hat{\gamma}_{i}\right\rangle$

(iv) two scalars $a_{i}, b_{i}$

denoting $\hat{\gamma}_{i}=\hat{\gamma}_{m=1, n(i)}$ and where $N_{\alpha}=N_{\tau} \neq N_{\hat{\gamma}}$. These datasets were accumulated from specialized T2R experiments as explained in subsection 1.5, and are presented as scatter-plots in figure 2. Index $j$ is essentially an enumeration of experiments such that we obtain one estimation of $\theta_{i} \forall i$ for each $j$. Estimation of $\tau_{i}^{(j)}$ and $\alpha_{i}^{(j)}$ is strongly interdependent and is depicted in figure 2(b), where the red circles are the arithmetic means. Figure 2(a) shows the estimated growth-rates and the thick lines 
shows arithmetic mean values. As can be seen by the mapping $n(i)$ this implies two growth-rates for each considered $n$ corresponding to spatial fourier mode $n(i)$ real part (black) and imaginary part (blue) respectively. This is interpreted as different phaselocations of otherwise similar perturbations. The fact that the black and blue curves do not coincide is in part plausibly due to the asymmetries of the shell. Horizontal and vertical cuts in the resistive shell imply that eddy currents are constrained in a phasedependent way, seen in spatial fourier domain. The estimates of parameters $a_{i}, b_{i}$ have been obtained as an average over multiple experiments (and therefore do not carry superscript $j$ ) by considering the control system's nearly steady-state compensation currents required for IS operation. As was suggested above, equation (8) is a model of the frequency content of this compensation and so $a_{i}, b_{i}$ expressly define the integrating requirements of the controllers in section 2 .

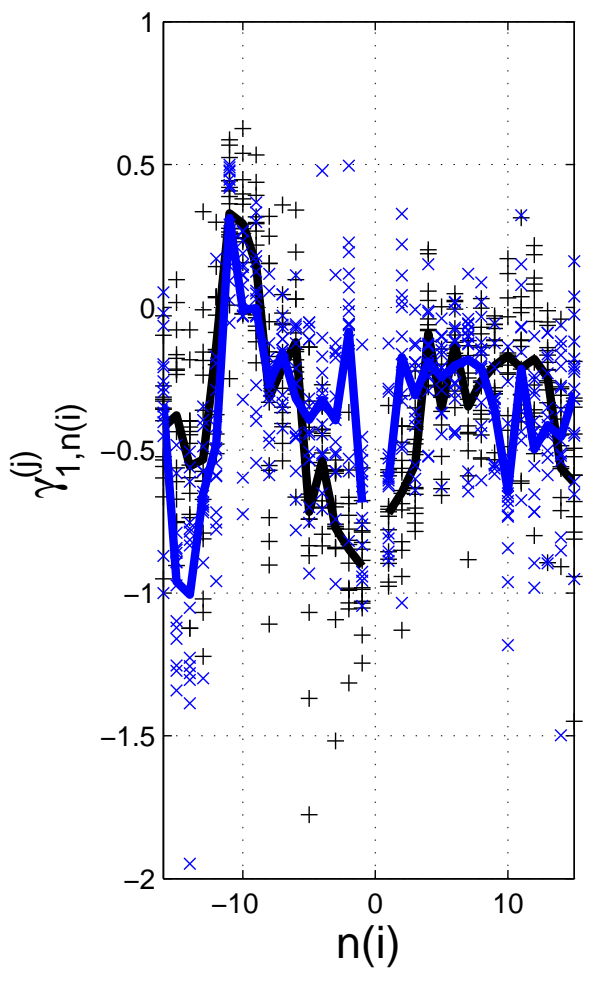

(a) Growth-rates $\hat{\gamma}_{1, n(i)}^{(j)}$ corresponding to real (black) and imaginary (blue) fourier component.

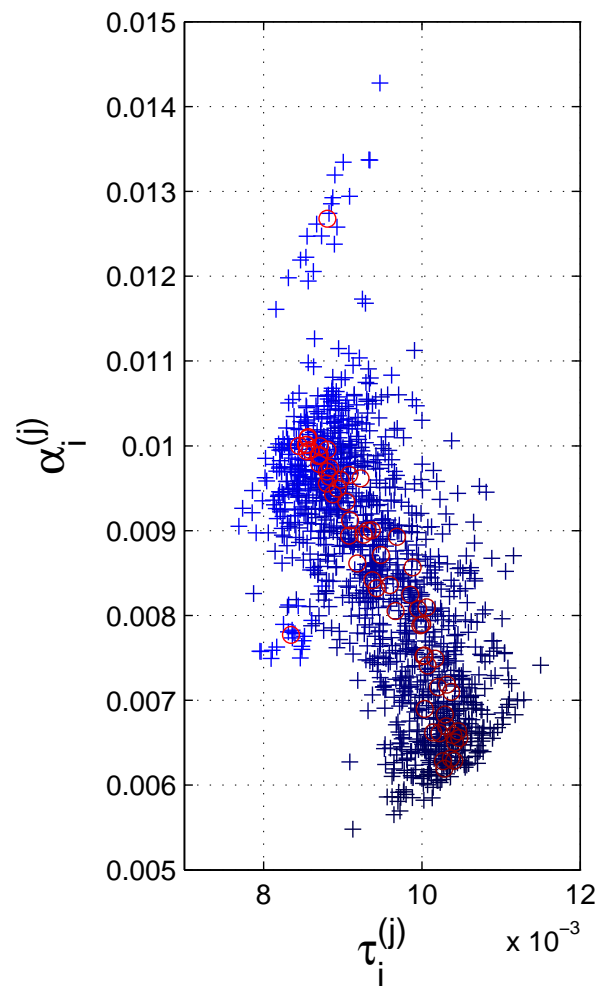

(b) Vacuum parameters scatterplot. Red circles are respective fourier modes' averages.

Figure 2. Estimated parameters from external plasma response identification experiment campaigns at $\mathrm{T} 2 \mathrm{R}$.

Fourier component single-input-single-output (SISO) controller synthesis (section 2 ) is solely based on the arithmetic mean parameters, while the robustness of the thus synthesised controllers are evaluated over the entire respective parameter sets above 
(subsection 3.1).

\subsection{Closed-loop identification and multivariable dithering}

Dedicated experiments $[5,6]$ at $\mathrm{T} 2 \mathrm{R}$ with pseudorandom [20] perturbations to the vector output of the IS control system (2) were conducted to gather data for the computations visualized in figure 2. Specifically the feedback system

$$
\tilde{\mathbf{u}}=M_{e q}\left(F_{P I D}(s)(\tilde{\mathbf{r}}-\tilde{\mathbf{y}})+\tilde{\mathbf{w}}\right)
$$

successfully stabilized the RWMs while simultaneously exciting spatial plasma modes. The axisymmetric set-point of $(10)$ was put $\tilde{\mathbf{r}}(t)=0$ together with perturbations on (dithering) auxiliary vector input $\tilde{\mathbf{w}}(t)$.

\section{Discrete-time fixed-order synthesis}

Here is the backdrop of this section: we have acquired a set of parameter values summarizing the external plasma response of $\mathrm{T} 2 \mathrm{R}$, subsection 1.4, and we have quantified the active amplifier response [17, 3]. There is a fast decoupling operation available (the FFT). The task at hand is to synthesize a bundle of digital controllers, one for each of the 64 FFT-components, that achieves 'good' closed-loop performance. Figure 3 illustrates the synthesis problem: devise a feedback controller $K$.

\subsection{Expressing and achieving closed-loop performance}

Given a SISO system in standard form

$$
\begin{aligned}
\mathbf{x}(k+1) & =A \mathbf{x}(k)+B u(k)+N v_{1}(k) \\
y(k) & =C \mathbf{x}(k)+v_{2}(k) \\
y_{m=1}(k) & =M \mathbf{x}(k)
\end{aligned}
$$

the objective here is to search for a fixed-order controller

$$
\begin{aligned}
\mathbf{x}_{K}(k+1) & =A_{K} \mathbf{x}_{K}(k)+B_{K} y(k) \\
u(k) & =C_{K} \mathbf{x}_{K}(k)+D_{K} y(k)
\end{aligned}
$$

that minimizes some functional of the resulting closed-loop system. Order $n_{K}=$ $\operatorname{dim} \mathbf{x}_{K}$ is given, not by the plant order but by the control system designer. As an additional constraint, the controller should not only stabilize the closed-loop but also be stable itself. This is known as strong stabilization and is beneficial in case of e.g. sensor failures.

Signals $u, y, y_{m=1}, v_{1}$ and $v_{2}$ in system (11) respectively correspond to actuator amplifier input, time-integrated sensor coil voltage measurement, the part of $y$ attributed to the $m=1$ mode, the driving process noise for the error-field, and finally the measurement noise.

Merging (11) and (12) and denoting the disturbance-inport $\mathbf{w}^{T}=\left(\begin{array}{ll}v_{1} & v_{2}\end{array}\right)$ and the performance-outport $\mathbf{z}^{T}=\left(\begin{array}{ccc}u & y & y_{m=1}\end{array}\right)$ yields

$$
\begin{aligned}
\overline{\mathbf{x}}(k+1) & =\mathcal{A} \overline{\mathbf{x}}(k)+\mathcal{B} \mathbf{w}(k) \\
\mathbf{z}(k) & =\mathcal{C} \overline{\mathbf{x}}(k)+\mathcal{D} \mathbf{w}(k)
\end{aligned}
$$


with augmented state $\overline{\mathbf{x}}^{T}=\left(\begin{array}{ll}\mathbf{x}^{T} & \mathbf{x}_{K}^{T}\end{array}\right)$ and matrices

$$
\begin{aligned}
& \mathcal{A}=\left(\begin{array}{cc}
A+B D_{K} C & B C_{K} \\
B_{K} C & A_{K}
\end{array}\right), \mathcal{B}=\left(\begin{array}{cc}
N & B D_{K} \\
0 & B_{K}
\end{array}\right) \\
& \mathcal{C}=\left(\begin{array}{cc}
D_{K} C & C_{K} \\
C & 0 \\
M & 0
\end{array}\right), \mathcal{D}=\left(\begin{array}{cc}
0 & D_{K} \\
0 & 1 \\
0 & 0
\end{array}\right)
\end{aligned}
$$

The transfer matrix associated with (13) is written

$$
H(z)=\mathcal{C}(z I-\mathcal{A})^{-1} \mathcal{B}+\mathcal{D}
$$

where $I$ is the identity matrix. If (13) is stable then solving the discrete-time Lyapunov equation [15]

$$
P_{x}=\mathcal{A} P_{x} \mathcal{A}^{T}+\mathcal{B} P_{w} \mathcal{B}^{T}
$$

for $P_{x}=\mathrm{E} \overline{\mathbf{x}}(k) \overline{\mathbf{x}}(k)^{T}$, where $P_{w}=\mathrm{Ew}(k) \mathbf{w}(k)^{T}$ and $\mathrm{E}$ the expectation operator, allows for the computation of the weighted matrix trace expression

$$
\operatorname{tr} P_{z} V_{z}
$$

with $V_{z} \geq 0$. The value of (18) is the central piece of the objective for the optimization program of subsection 2.3. The output-covariance matrix $P_{z}$ is computed from the Lyapunov solution $P_{x}$ as

$$
P_{z}=\operatorname{Ez} \mathbf{z}(k) \mathbf{z}(k)^{T}=\mathcal{C} P_{x} \mathcal{C}^{T}+\mathcal{D} P_{w} \mathcal{D}^{T}
$$

Two particular weights of the trace (18) are considered here $(q \geq 0)$

$$
V_{z}^{(i)}=\left(\begin{array}{ccc}
q & 0 & 0 \\
0 & 1 & 0 \\
0 & 0 & 0
\end{array}\right), \quad V_{z}^{(i i)}=\left(\begin{array}{ccc}
q & 0 & 0 \\
0 & 0 & 0 \\
0 & 0 & 1
\end{array}\right)
$$

to distinguish between (i) IS-like and (ii) CMC-like controllers: subsection 2.4.

The extension to tracking is readily achieved by substitution of compensator input $y(t)$ with $y(t)+\operatorname{lr}(t)$ where $l$ is a scalar constant defined by the steady-state gain as follows.

$$
\begin{aligned}
& l^{(i)}=\left\{\left(\begin{array}{ll}
C & 0
\end{array}\right)(I-\mathcal{A})^{-1}\left(\begin{array}{c}
B D_{K} \\
B_{K}
\end{array}\right)\right\}^{-1} \\
& l^{(i i)}=\left\{\left(\begin{array}{ll}
M & 0
\end{array}\right)(I-\mathcal{A})^{-1}\left(\begin{array}{c}
B D_{K} \\
B_{K}
\end{array}\right)\right\}^{-1}
\end{aligned}
$$

Superscript indexing in (21) corresponds to the similarly indexed doublette in (20).

Now, having introduced the required notational bulk above, we provide a common interpretation of objective (18). If $v_{1}$ and $v_{2}$ are white sequences with covariance $P_{w}$ then the output covariance of the closed-loop is $P_{z}$, hence the weighting defined by (20) translates to a sum of variances. This is indeed parallel to standard LQG-synthesis $[15,24]$, the main challenge here being the fixed-order requirement. 


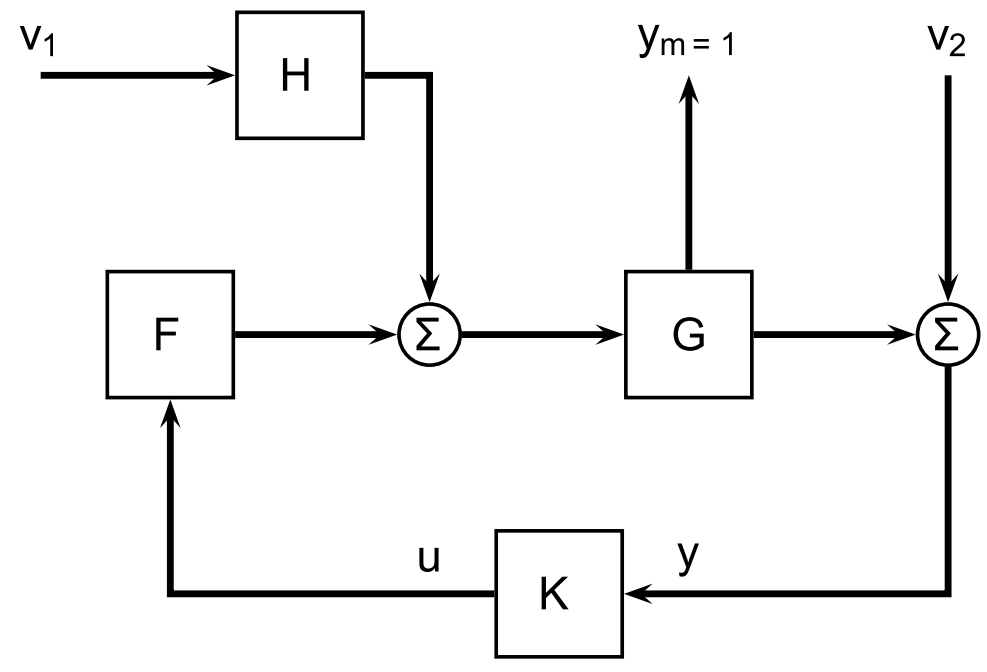

Figure 3. Signal model for closed-loop synthesis.

\subsection{Instantiation of system matrices for T2R}

The signal routing of figure 3 with T2R-particular incarnations of $F, G$ and $H$ need to be expressed in the form (11) prior to compilation of (14)-(15). The open-loop version of figure 3, i.e. controller $K$ removed leaving $u$ and $y$ as inport and outport respectively, in format (11) is immediately given by

$$
\begin{aligned}
A & =\left(\begin{array}{ccc}
A_{F} & 0 & 0 \\
0 & A_{H} & 0 \\
B_{G} C_{F} & B_{G} C_{H} & A_{G}
\end{array}\right) \\
B & =\left(\begin{array}{c}
B_{F} \\
0 \\
0
\end{array}\right), N=\left(\begin{array}{c}
0 \\
B_{H} \\
0
\end{array}\right) \\
C & =\left(\begin{array}{ccc}
0 & 0 & C_{G}
\end{array}\right), M=\left(\begin{array}{lll}
0 & 0 & M_{G}
\end{array}\right)
\end{aligned}
$$

for the state $\mathbf{x}=\left(\begin{array}{lll}\mathbf{x}_{F}^{T} & \mathbf{x}_{H}^{T} & \mathbf{x}_{G}^{T}\end{array}\right)^{T}$. System matrices $\left(A_{G}, B_{G}, C_{G}, M_{G}\right)$ for $G$ are derived from (5), (6) and (7). System $F$ is constructed by zero-order-hold discretization $F^{\prime}(z)$ of the continuous-time representation of the bundled amplifier plus coil model $F^{\prime}(s)=\frac{k_{F}}{\tau_{F} s+1}$, where $k_{F}=4.1$ and $\tau_{F}=1.1 \times 10^{-3} \mathrm{~s}$. A onesample delay augmentation to account for computational latency $[19,7]$ then defines actuation-block $F(z)=z^{-1} F^{\prime}(z)$. The state space equivalent of $F(z)$ is $\left(A_{F}, B_{F}, C_{F}\right)$. Equation (8) implicitly defines $\left(A_{H}, B_{H}, C_{H}\right)$. From eyeballing the above information it follows that $\operatorname{dim} \mathbf{x}_{F}=2, \operatorname{dim} \mathbf{x}_{H}=1$, and $\operatorname{dim} \mathbf{x}_{G}=9$. Zero entries in (23) are dimensioned according to respective state size.

\subsection{Optimization program}

An unconstrained nonlinear optimization program is readily distilled from the material in the preceeding sections. This is achieved by relaxation of the two stability constraints, which emerge as penalty terms in the merit function. We seek a solution 
to

$$
\min _{K \in \mathbb{R}^{\left(n_{K}+1\right) \times\left(n_{K}+1\right)}} f(K)
$$

where

$$
f(K)=c_{1} \operatorname{tr}\left(P_{z}(K) V_{z}\right)+c_{2} \mathbf{p}^{T}(K) \Lambda \mathbf{p}(K)
$$

with

$$
\begin{aligned}
& K=\left(\begin{array}{ll}
A_{K} & B_{K} \\
C_{K} & D_{K}
\end{array}\right) \\
& \mathbf{p}(K)=\left(\begin{array}{c}
\max \left(0, \max _{i}\left|\lambda_{i}(\mathcal{A}(K))\right|-1\right) \\
\max \left(0, \max _{i}\left|\lambda_{i}\left(A_{K}(K)\right)\right|-1\right)
\end{array}\right)
\end{aligned}
$$

and $\Lambda \geq 0$. The notation $\lambda_{i}(M)$ for a matrix $M$ represents the $i$ th eigenvalue of the matrix. Program (27) is in general nonconvex and highly local suboptimum solutions must be defenestrated. Note that the classical change of variables that would allow for a linear formulation of the optimization problem [25] typically implies that the controller rank is equal to the system rank and consequently cannot be applied here. A two-step procedure has been devised for (26), (27) as explained in the next paragraph.

For the first stage: randomize a $K=K^{(0)}$ and put $c_{1}=0$ and $c_{2}=1$ and simply search for any $K$ having zero merit value (zero penalty). Then put $c_{1}=1$ and $c_{2}=10^{3}$ and resume the minimization (now including the trace merit). See reference [26] for more sophistication regarding fixed-order strong stabilization synthesis. Standard BFGS $\ddagger$ method $[27,20]$ with numeric finite differences has been employed and leads to satisfactory results. The synthesis proceeds by randomly sampling initial controllers for a preset maximum number of repetitions $n_{\max }$. In this case $n_{\max }=30$ proved sufficient, given sampling around a $K^{(0)}$ representing a blindly tuned nominal PID as detailed in [3] and recapitulated below. For this study $q=10^{-6}$ in (20) and $\Lambda=I$ in (27) were chosen.

The reason for first finding a stable and stabilizing $K$ without regard for the trace term is that the trace term only makes sense if the system is already stable. For unstable or marginally unstable systems the Lyapunov equation might have no solution or a solution does not have to be positive. Having computed a valid trace term in the stabilizing/stable region we try to stay there by severly penalizing any exit from this region, while trying to minimize the trace term according to (27).

Due to the nonuniqueness of state-space system realizations, there can be no hope to relapse into the same optimal parameter-value $K^{\star}$. Specifically, an arbitrary statespace system (state $x$ ) representation $K_{1}=\left(\begin{array}{cc}A & B \\ C & D\end{array}\right)$ is equivalent to the coordinatetransformed (state $x^{\prime}$ ) system $K_{2}=\left(\begin{array}{cc}T A T^{-1} & T B \\ C T^{-1} & D\end{array}\right)$ for any $|T| \neq 0, x^{\prime}=T x . K_{1}$ and $K_{2}$ are numerically different $\left(K_{1} \neq K_{2}\right)$ realizations $\oint$ of the same system. The synthesis algorithm will consequently, at best, produce different realizations of the same dynamical system; by relapsing to the same (locally) minimum objective value.

Finally, the first-stage randomization was chosen as follows.

$$
K^{(0)}=K_{C}+\rho R_{\rho}
$$

$\ddagger$ Broyden-Fletcher-Goldfarb-Shanno

$\S$ It is sometimes favourable to seek some specific optimal realization, e.g. for numerical stability of an implementation. 
where $\rho=0.1, R_{\rho} \in \mathbb{R}^{\left(n_{K}+1\right) \times\left(n_{K}+1\right)}$ with each matrix element a scalar independently sampled from a normal distribution with zero mean and unit variance, and $K_{C}$ a zero-order-hold discretization of the PID controller

$$
C(s)=k_{p}+\frac{k_{i}}{s}+\frac{k_{d} s}{k_{d} s / N_{d}+1}
$$

with $k_{p}=40, k_{i}=5000, k_{d}=0.04$ and $N_{d}=30$. In this case, $n_{K_{C}}=2$.

\subsection{Conformed nomenclature of synthesis flavours}

As hinted above, the search for minima to (26) is performed in two different ways as follows.

IS-like : Solve (26) using $V_{z}^{(i)}$ of (20) and implement with scalar $l^{(i)}$ in (21) for reference term.

CMC-like : Solve (26) using $V_{z}^{(i i)}$ of (20) and implement with scalar $l^{(i i)}$ in (21) for reference term.

The reason for introducing the bold-fonted adjectives above is to relate and align to notations already encountered in the literature. IS-like synthesis is also known as output-tracking [3] and can be equated to a generalised, but still classic, intelligent-shell [4]. The aim for IS-synthesised feedback systems is to control the measured output directly, i.e. the averaged radial magnetic field components precisely incident at the sensor array. In contrast, the CMC-like controller attempts to relate this measured output to a fictitious output of a model, as encompassed by (5), with the ambition of tracking an inferred dynamic state; the $y_{m=1}$ performance output of (13). The acronym CMC is attached to this idea as concocted in [10]. The central problem addressed by $\mathrm{CMC}$ is spatial aliasing due to finite sensor and actuator arrays. In this work we use an experimentally identified plasma reponse model and quadratic cost minimization of a closed-loop performance-channel while [10] proceeds by designing a basic spatial filter prior to a manually tunable proportional feedback control with no explicit considerations of the full closed-loop system properties. However, in [10], versatile expressions for extrapolation of radial magnetic field for radial positions not incident at the sensor array are developed. This could also be included in the design presented here, but is a topic for further work.

\section{Synthesis results and implementational aspects}

There are two topics of this section. First, statistical issues and robustness of the synthesis described in section 2 are addressed, and second, some information on the real-time implementational details is provided, hopefully shedding light on why the fixed-order controller constraint is imperative for T2R.

\subsection{Postsynthesis bootstrap worst-case gain}

The synthesis of section 2 handled a single system. Before allowing such a controller to work on the plasma, it is desirable that it might robustly handle the variations in the identification data sets. In order to evaluate some robust performance it is then resonable to somehow the predict closed-loop behaviour using all the available data points. 
The worst-case gain for any stable system $(A, B, C, D)$ can be computed by solving the convex program

$$
\text { s.t. }\left\{\begin{array}{cc}
\min _{X, \gamma} \gamma \\
\left(\begin{array}{cc}
-X+A^{T} X A+C^{T} C & A^{T} X B+C^{T} D \\
B^{T} X A+D^{T} C & B^{T} X B-\gamma^{2}+D^{T} D
\end{array}\right)<0 \\
X=X^{T}>0
\end{array}\right.
$$

where the LMI $\|$-constraints emerge by applying the discrete-time bounded-real lemma [28]. The unique solution $\gamma^{*}$ to (32) is then the minimum $\gamma$ fulfilling

$$
\forall z \in \mathbb{C},|z|=1: \bar{\sigma}\left(C(z I-A)^{-1} B+D\right) \leq \gamma
$$

i.e. the worst-case gain bound over frequency for discrete-time systems. Program (32) is infeasible if $A$ has eigenvalues outside or on the unit circle and $\bar{\sigma}$ extracts the largest singular value $[15,20]$ of a matrix.

We wish to estimate the expected closed-loop behaviour over the full identification dataset for each fourier component dynamics. This verifies the robustness of the synthesised controllers, with respect to available parameter data only. It can be done thanks to bootstrap computation [29] of the worst-case gain distribution of the closedloop sensitivity transfer function. This proceeds as follows.

Randomly select three parameters $\alpha_{i}, \tau_{i}, \hat{\gamma}_{i}$ uniformly from the datasets in subsection 1.4. Then assemble the $i$ th closed-loop system matrices, using the controller synthesised for component $i$, and recall from (16) $\mathcal{H}(z)$. Put $T_{y w}(\omega)=$ $\left(\begin{array}{lll}0 & 1 & 0\end{array}\right) \mathcal{H}\left(e^{\iota \omega}\right)$ and find minimal $\gamma^{*}$ such that $\forall \omega \in \mathbb{R}: \max _{\omega} \bar{\sigma}\left(T_{y w}(\omega)\right) \leq \gamma^{*}$ by solving (32) with $(A, B, C, D)$ representing $T_{y w}$. Repeat this $N_{b o o t}$ times and the set of $\gamma^{*}$ so obtained suggests what induced closed-loop performance variations can be expected from the scattered parameters of figure 2 .

The bootstrap histogram of $\gamma^{*}$ for $i=28(n(28)=-5$, real part $)$ is presented in figure 4(a), where $N_{b o o t}=5 \times 10^{4}$. The minimum and maximum worst-case gains together with a random system sample of $\bar{\sigma} T_{y w}$ for these simulations can be viewed in figure 4(b). The results for this particular fourier component is representative for the entire range of $i=1 \ldots 64$. In conclusion, the synthesised controllers supposedly should behave robustly over the uncertain system data of subsection 1.4. This postsynthesis certification is indeed very general, but we are here content with a confirmation of un-dramatic $\mathcal{H}_{\infty}$-performance. As should be clear from (32) a stability-check is implicit in this procedure.

\subsection{Real-time implementational tricks}

As stated in the introduction, we are at the moment confined to use hardwareaccelerated [21] FFT-decoupling. CPU 9 -cycle spendthriftness is, at the time of writing, unacceptable at T2R. To gobble controller coefficients and magnetic measurements into control output at the required rate in silico we have been forced to $\mathrm{SIMD}^{+}$-specific programming tricks-of-the-trade. Storing the system coefficients synthesised above with some precaution enables controller state and output to be updated using only three vector multiply-accumulate [30] operations for $n_{K}=2$ as detailed below.

\| Linear matrix inequality

I Central processing unit

+ Single instruction multiple data 


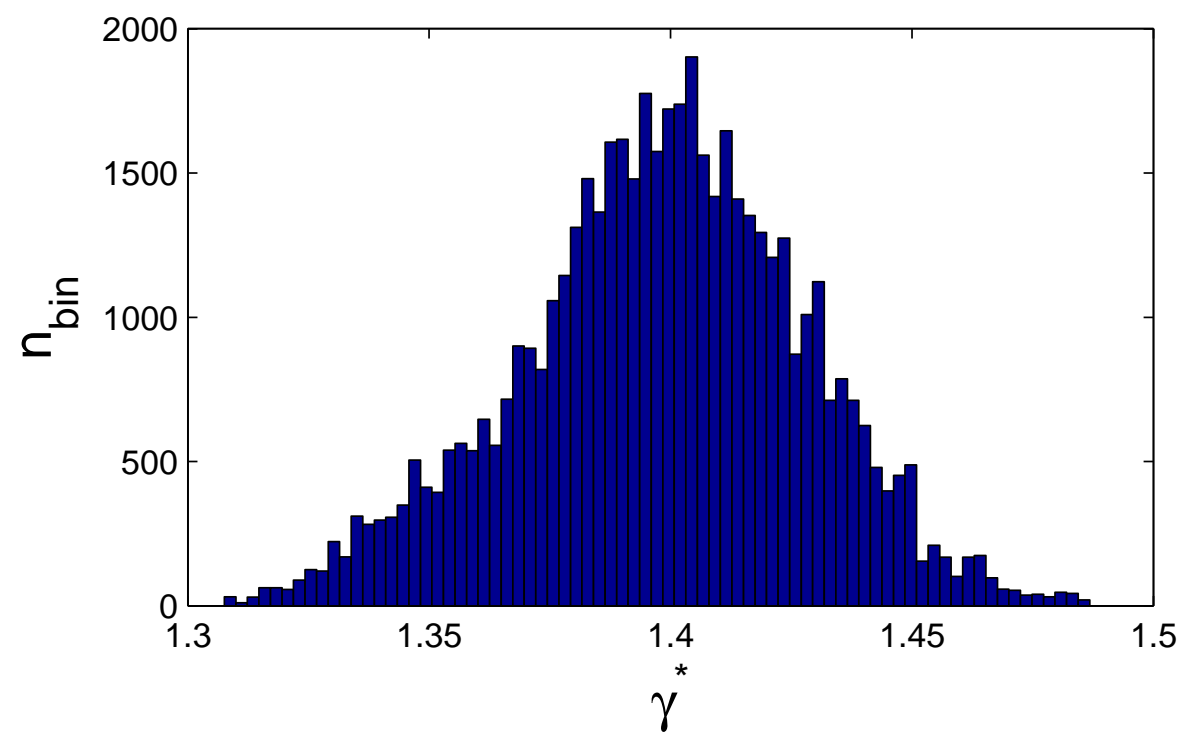

(a) Bootstrap distribution of $\gamma^{*}$

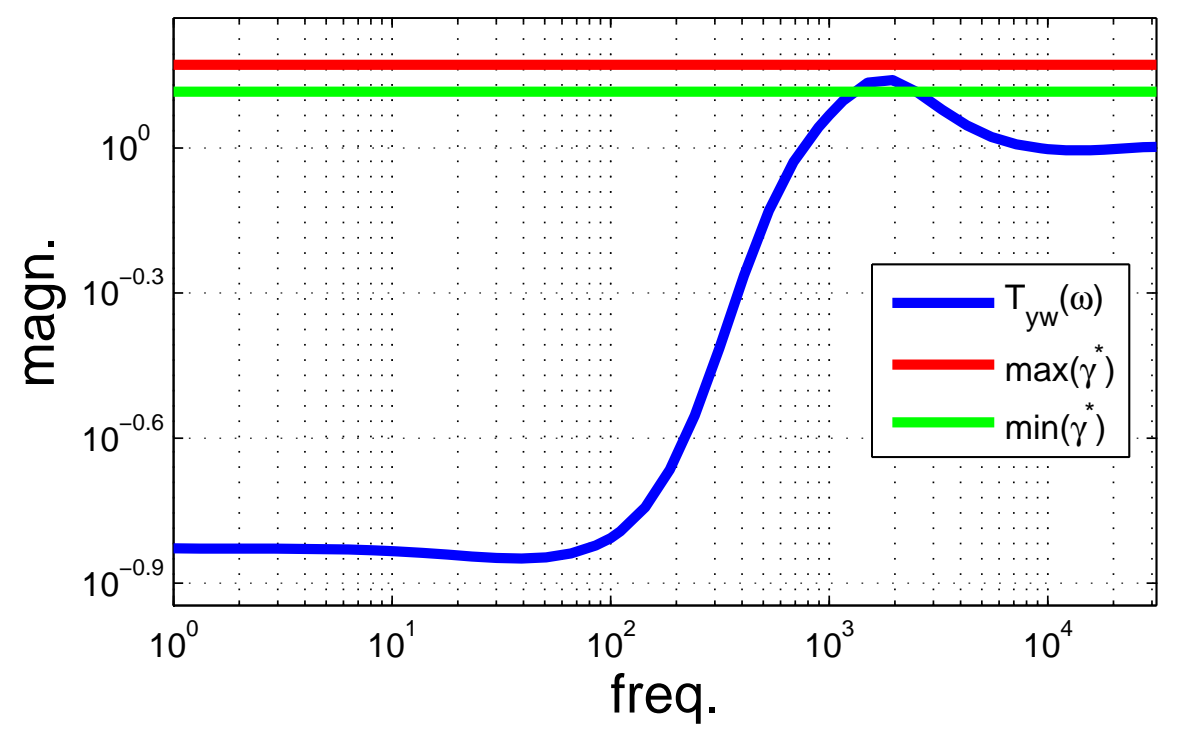

(b) Randomly sampled sensitivity function $T_{y w}(\omega)$ (blue) and $\min / \max$ worst-case gains over bootstrap computation (green/red).

Figure 4. Bootstrap simulation, $N_{\text {boot }}=5 \times 10^{4}$, of closed-loop worst-case gain distribution over identification data sets. 


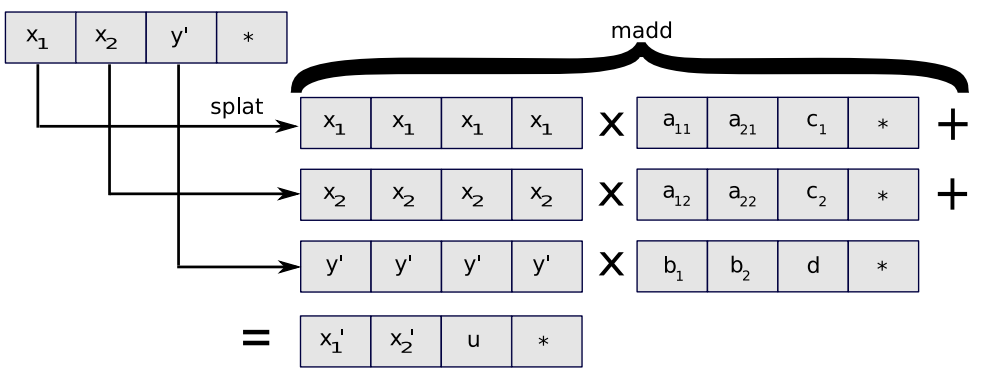

Figure 5. Vector register splat and madd SIMD-operations illustrated.

We document this in (idiosynchratic) detail, figure 5, to emphasize the importance of the fixed-order requirement. Apparently, if states, inputs and outputs can be fitted within a single vector register of $4 \times$ floating-point single-precision numbers (32-bit each), then the actual number of executed CPU cycles can be greatly reduced. Indeed, the splat and the madd (multiply and accumulate) instructions, with necessary interstitial loads provide a rapid execution of parallel state update and output computation. In figure 5 , the input register is $\left(x_{1}(k) \quad x_{2}(k) \quad y^{\prime}=y(k)+\operatorname{lr}(k) \quad *\right)$, the output is

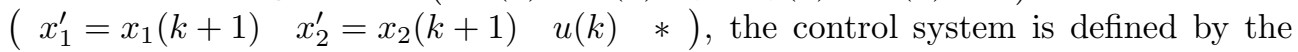
system matrix $K=\left(\begin{array}{ccc}a_{11} & a_{12} & b_{1} \\ a_{21} & a_{22} & b_{2} \\ c_{1} & c_{2} & d\end{array}\right)$ and finally $*$ means "unspecified". Note that the matrix coefficients are stored pretransposed and aligned in memory such that load and store overhead is minimal.

It is seen, from figure 5 , that any $n_{K}=3$-controller can be implemented by merely repeating one more iteration of splat/madd: a very small extra computational time. In this study, only $n_{K}=2$ controllers have been synthesised and operated.

\section{Experimental results}

First plasma operation with the digital controller developed here went smoothly. T2R was stabilized using the new controller reconfiguration and we can declare a 'first-pass system success' in this respect.

In order to investigate the experimental behaviours of the different designs, i.e. IS- and CMC-like respectively, five shots were performed for each of the synthesised sets of coefficents. The expected behaviour is that the $y_{m=1}$, or CMC-like controller, will be slightly worse at supressing the plant output compared to the $y$-synthesis (which is exactly designed to do plant output minimization). Indeed, we can see this particular relation in the experimental data, as presented in table 2. Output-energy seems consistently larger for the CMC-like design. Admittedly, five shots is a very weak statistical basis for strong claims, unless the plant is largely deterministic. Under the assumption of applicability of the underlying linear model (4) this is however a firm indication that the reconfigured control system was implemented correctly.

Definition of energy measure quoted in table 2 is

$$
P_{y, n}=\frac{1}{k_{2}-k_{1}+1} \sum_{k=k_{1}}^{k_{2}}\left|y_{n}(k)\right|^{2}
$$


Table 2. Summary of average measured field incident at the sensor array in interval $t \in[10,70] \mathrm{ms}$. Five shots each for the two different synthesis cases.

\begin{tabular}{c|c|c|c} 
synthesis & shot-number & $P_{y}\left[(\mathrm{mT})^{2} \times 10^{-3}\right]$ & $\left\langle P_{y}\right\rangle$ \\
\hline \multirow{5}{*}{$y$} & 21821 & 2.80 & \\
& 21825 & 3.20 & \\
& 21826 & 3.42 & 3.23 \\
& 21827 & 3.23 & \\
$y_{m=1}$ & 21828 & 3.50 & \\
& 21829 & 3.55 & \\
& 21830 & 3.98 & 3.68 \\
& 21831 & 3.86 &
\end{tabular}

$$
P_{y}=\sum_{i \in\{1, \ldots 32\}, n(i) \neq 0} P_{y, n(i)}
$$

using a notation

$$
y_{n}=(\mathcal{T} \tilde{\mathbf{y}})_{i} f_{y}, \text { such that } n=n(i)
$$

and with $k_{1}$ and $k_{2}$ denoting the first and last sample indices corresponding to the true time interval of interest. The bracket-notation $\langle\cdot\rangle$ in header of table 2 takes arithmetic average over the table row subset. The scalar multiplier $f_{y}$ in (36) converts the measurements to units of millitesla $[\mathrm{mT}]$.

Tracking capabilities were also verified experimentally and are presented here with two examples in figure 6 . The first, figure 6(a), is an on-and-off toggled sequence of assorted spatial fourier modes applied as a sustained stationary-phase boundary condition. The second shows two spatial fourier modes with simultaneous phaserotation as a dynamic sustained boundary; figure 6(b). Both these examples were enforced by running the CMC-like controller.

The shot in figure 6(b) deserves a companion visual. Figure 7 clearly shows the rotating boundary field consisting of two fourier components $n=5$ and $n=1$. Vertical black dashed lines bracket the duration of the plasma and white horizontal line separates the toroidally sorted groups of measurement channels. Horizontal field displacement being $i=1 \ldots 32$ and vertical $i=33 \ldots 64$. Scrutinizing figures $6(\mathrm{~b})$ and 7 it can be seen that the two concurrently tracked fourier modes have equal phasevelocity magnitude $(25 \mathrm{~Hz})$, but with opposite signs. To the best of the authors' knowledge this clean-looking stabilized dynamic boundary tracking is unparalleled by other toroidal plasma experiments, at the time of writing.

\section{Conclusion and outlook}

At T2R, this work is a breakthrough in several significant aspects. For the first time, we have synthesised a control system directly inferred from measured plasma response data. No ad hoc tampering with any control knob was required, the design ran out-ofthe-box. This is a point that should be emphasized. The general template for process control system development was adapted for the RFP: system identification followed 


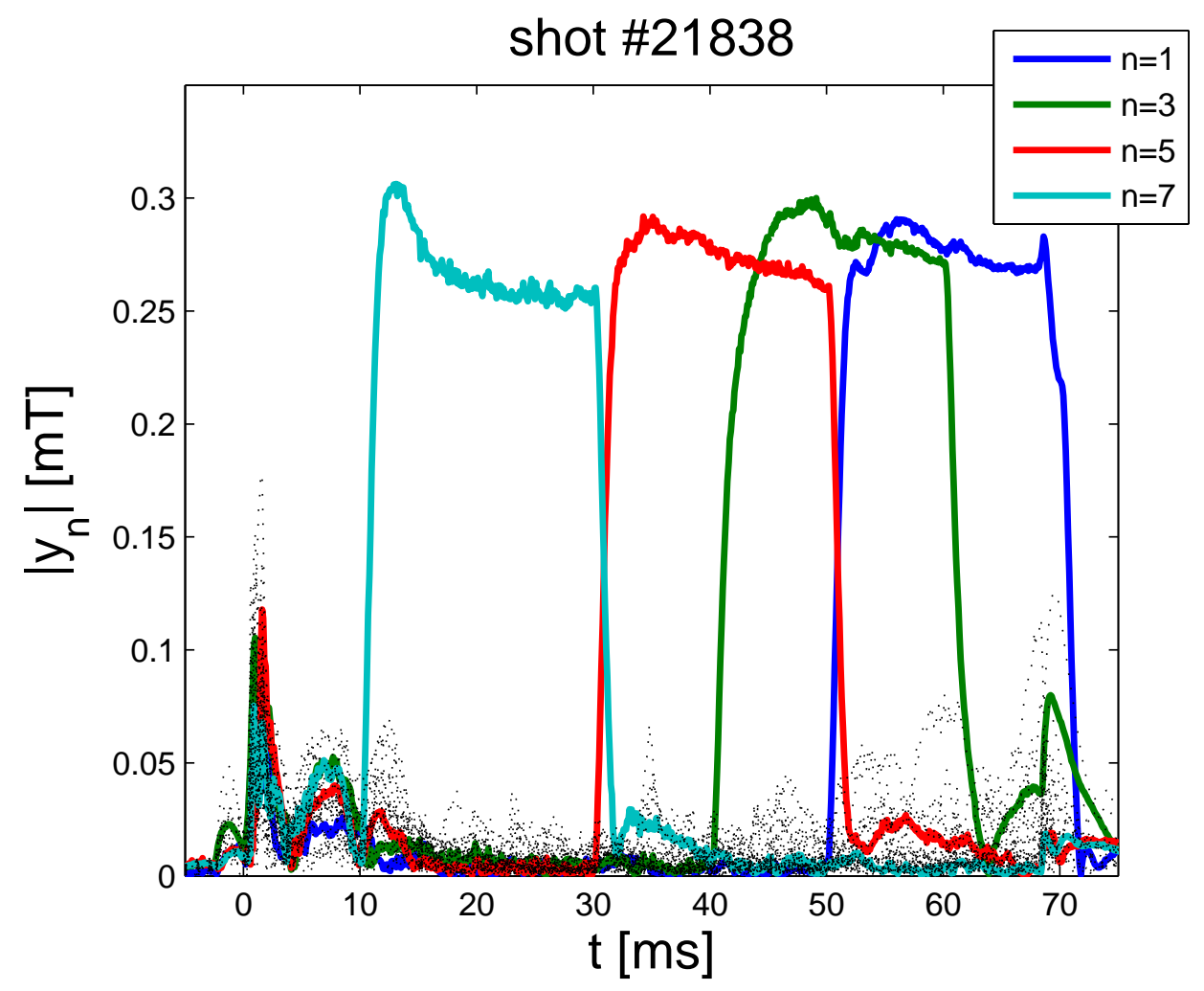

(a) Static-phase (overlapping) sequence of modes.

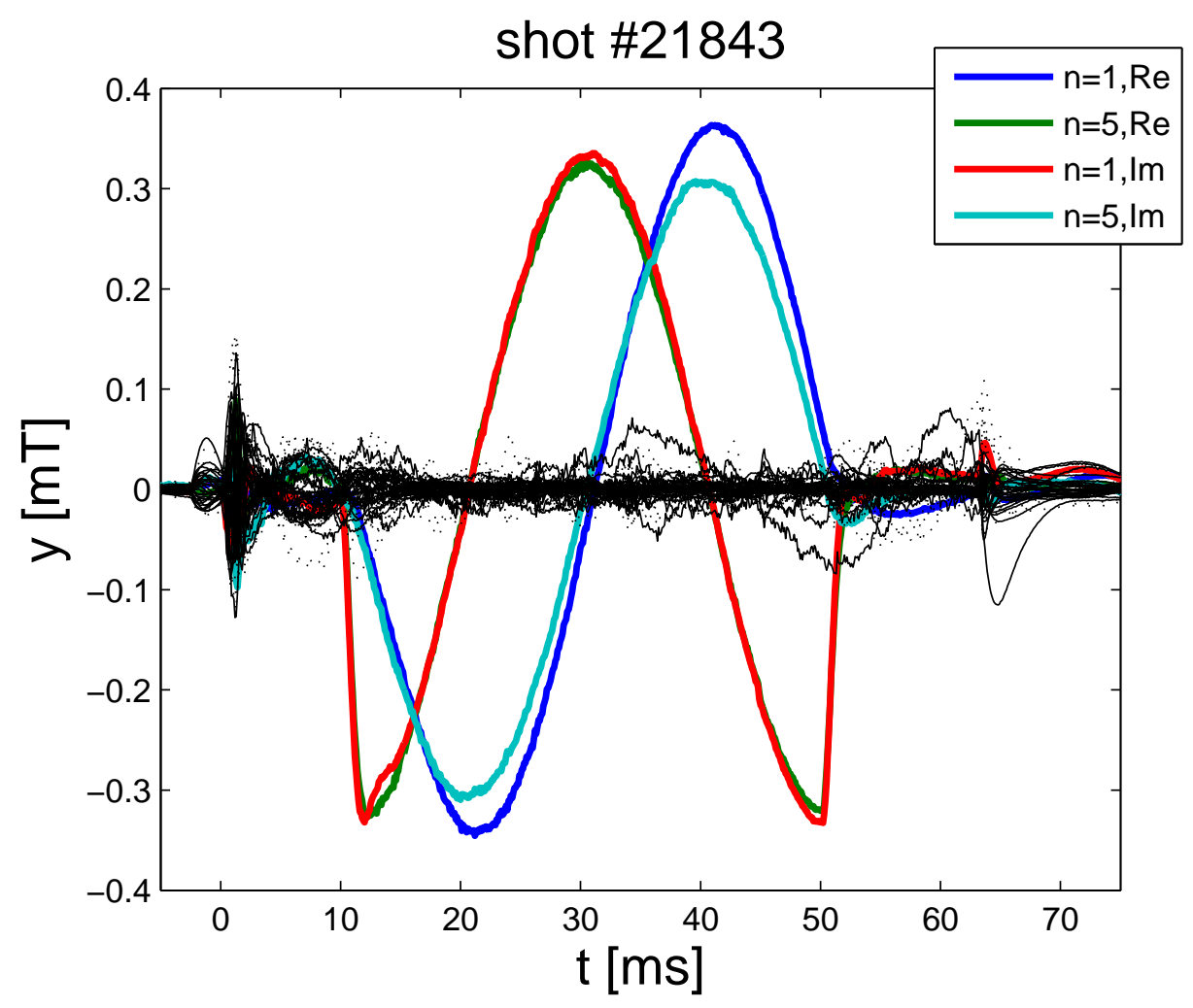

(b) Stabilized and sustained rotating boundary field.

Figure 6. Measurements from radial field sensor coils. 


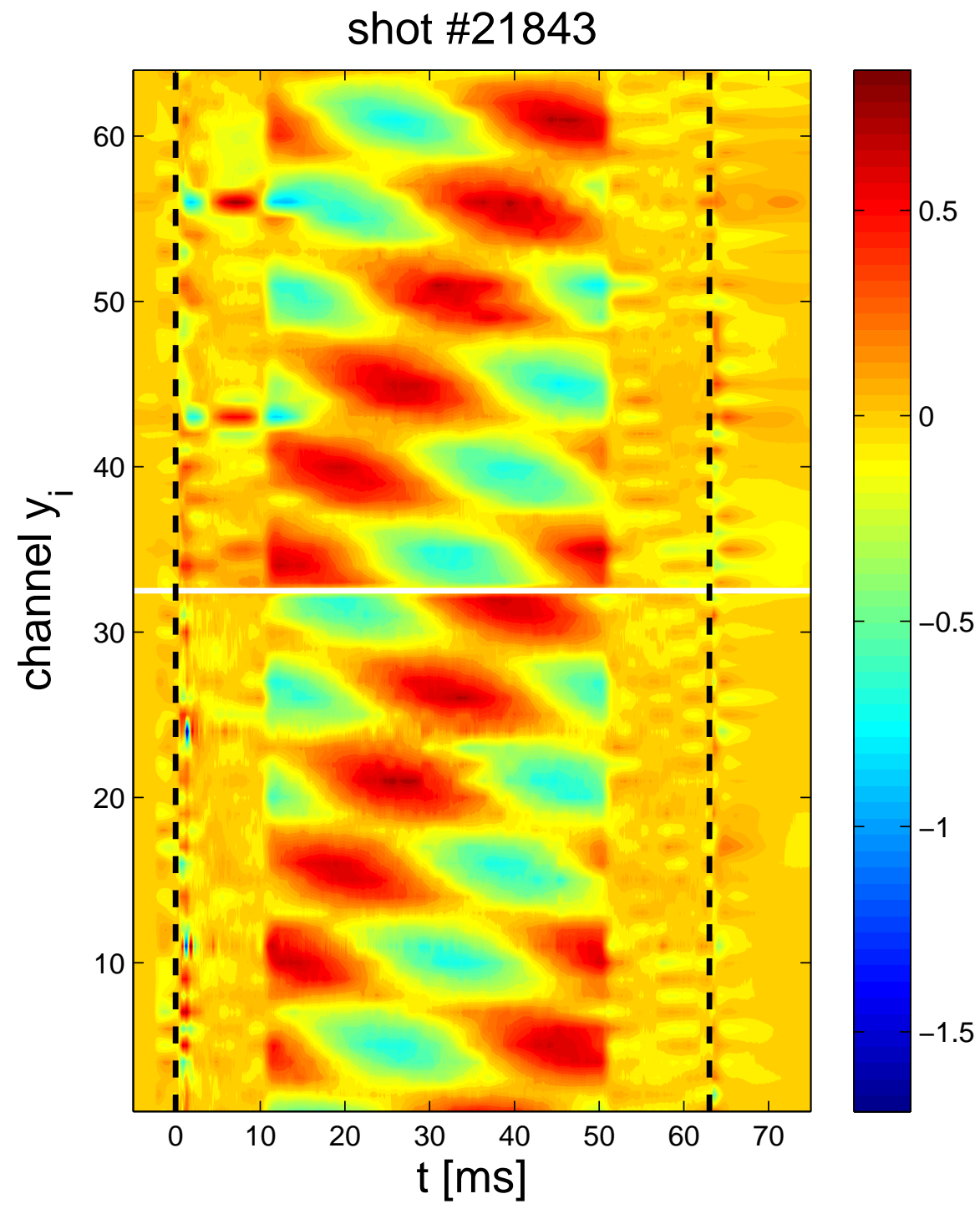

Figure 7. Toroidally sorted channel-space time-evolution picture of shot \#21843. 
by controller setup based on the identification results. We here showed the last stage of this procedure and deployed a control system that worked as it was supposed to, at the first shot.

A natural sequel to this study is to develop plasma response identification methods to relax the hitherto enforced clean fourier eigenmode structure. It can furthermore be physically interesting to carefully search for systematic differences in detailed plasma performance between the centralized designs presented here with the rehashed/refurbished decentralized IS-design (RIS) hatched in [3]. What can be immediately seen is that both [3] and the FFT-decoupled system developed here stabilize the plasma similarly, in the sense that the same shot-length is attained for the respective strategies.

\section{References}

[1] P. R. Brunsell et al. Feedback stabilization of multiple resistive wall modes. Physical Review Letters, 93(22):225001, 2004.

[2] D. Yadikin. Resistive wall mode stability and control in the reversed-field pinch. Doctoral thesis, KTH Electrical Engineering, Stockholm, Sweden, 2006.

[3] Erik Olofsson and Per Brunsell. Controlled magnetohydrodynamic mode sustainment in the reversed-field pinch: Theory, design and experiments. Fusion Engineering and Design, 84(711):1455-1459, 2009. Proceeding of the 25th Symposium on Fusion Technology - (SOFT-25).

[4] C.M. Bishop. An intelligent shell for the toroidal pinch. Plasma Physics and Controlled Fusion, 31(7):1179-1189, 1989.

[5] Erik Olofsson, Per Brunsell, and James Drake. Closed-loop direct parametric identification of magnetohydrodynamic normal modes spectra in EXTRAP-T2R reversed-field pinch. Proceedings of the 3rd IEEE Multi-conference on Systems and Control (MSC), Jul. 2009.

[6] Erik Olofsson, Håkan Hjalmarsson, Cristian Rojas, Per Brunsell, and James Drake. Vector dither experiment design and direct parametric identification of reversed-field pinch normal modes. Proceedings of the 48th IEEE Conference on Decision and Control (CDC), page to appear, Dec. 2009.

[7] P. R. Brunsell, K. E. J. Olofsson, L. Frassinetti, and J. R. Drake. Resistive wall mode feedback control in EXTRAP T2R with improved steady-state error and transient response. Physics of Plasmas, 14(10):102505, 2007.

[8] M. Okabayashi, N. Pomphrey, and R.E. Hatcher. Circuit equation formulation of resistive wall mode feedback stabilization schemes. Nuclear Fusion, 38(11):1607-1627, 1998.

[9] T. Sunn Pedersen, D.A. Maurer, J. Bialek, O. Katsuro-Hopkins, J.M. Hanson, M.E. Mauel, R. James, A. Klein, Y. Liu, and G.A. Navratil. Experiments and modelling of external kink mode control using modular internal feedback coils. Nuclear Fusion, 47(9):1293-1299, 2007.

[10] Paolo Zanca, Lionello Marrelli, Gabriele Manduchi, and Giuseppe Marchiori. Beyond the intelligent shell concept: the clean-mode-control. Nuclear Fusion, 47(11):1425-1436, 2007.

[11] A Portone, F Villone, Y Liu, R Albanese, and G Rubinacci. Linearly perturbed MHD equilibria and 3D eddy current coupling via the control surface method. Plasma Physics and Controlled Fusion, 50(8):085004 (12pp), 2008.

[12] P R Brunsell, H Bergsaker, M Cecconello, J R Drake, R M Gravestijn, and A Hedqvist ad2 and J-A Malmberg. Initial results from the rebuilt EXTRAP T2R RFP device. Plasma Physics and Controlled Fusion, 43(11):1457-1470, 2001.

[13] D. Gregoratto, J. R. Drake, D. Yadikin, Y. Q. Liu, R. Paccagnella, P. R. Brunsell, T. Bolzonella, G. Marchiori, and M. Cecconello. Studies on the response of resistive-wall modes to applied magnetic perturbations in the EXTRAP T2R reversed field pinch. Physics of Plasmas, 12(9):092510, 2005.

[14] R. Paccagnella, D. Gregoratto, and A. Bondeson. Feedback control of resistive wall modes in reversed field pinches. Nuclear Fusion, 42(9):1102-1109, 2002.

[15] Torkel Glad and Lennart Ljung. Control Theory: Multivariable and Nonlinear Methods. CRC, 1st edition, 2000.

[16] Gene F. Franklin, J. David Powell, and Michael L. Workman. Digital Control of Dynamic Systems. Prentice-Hall, 3rd edition, 1997.

[17] Darya Ivanova. Development of power amplifier model and feedback controller design for active 
MHD mode control at the EXTRAP-T2R reversed field pinch device. Master's thesis, Royal Institute of Technology (KTH), June 2008.

[18] Karl J. Åstrom and Tore Hägglund. PID Controllers: theory, design, and tuning. International Society for Measurement and Control, 2nd edition, 1995.

[19] E. Olofsson, E. Witrant, C. Briat, S.-I. Niculescu, and P. Brunsell. Stability analysis and modelbased control in EXTRAP-T2R with time-delay compensation. Proceedings of the 47 th IEEE Conference on Decision and Control (CDC), pages 2044-2049, Dec. 2008.

[20] W.H. Press, S.A. Teukolsky, W.T. Vetterling, and B.P. Flannery. Numerical Recipes in $C++$. Cambridge University Press, 2nd edition, 2002.

[21] AN2115. Complex floating-point fast fourier transform optimization for AltiVec ${ }^{\mathrm{TM}}$. Technical report, Freescale Semiconductor, Inc., October 2006.

[22] R. Fitzpatrick and E. P. Yu. Feedback stabilization of resistive shell modes in a reversed field pinch. Physics of Plasmas, 6(9):3536-3547, 1999.

[23] Lennart Ljung. System Identification: Theory for the User. Prentice Hall PTR, 2nd edition, 1999.

[24] Vikram Kapila and Wassim M Haddad. $H_{2}$ stable stabilization for discrete-time systems. Proceedings of the 34th IEEE Conference on Decision and Control (CDC), December 1995.

[25] Carsten Scherer, P. Gahinet, and M. Chilali. Multiobjective output-feedback control via LMI optimization. IEEE Transactions on Automatic Control, 42(7):896-911, Jul 1997.

[26] S. Gumussoy, M. Millstone, and M.L. Overton. H- $\infty$ strong stabilization via HIFOO, a package for fixed-order controller design. Proceedings of the 47 th IEEE Conference on Decision and Control (CDC), pages 4135-4140, Dec. 2008.

[27] David G. Luenberger. Linear and nonlinear programming. Kluwer Academic Publishers, 2nd edition, 2003.

[28] Stephen Boyd, Laurent El Ghaoui, E. Feron, and V. Balakrishnan. Linear Matrix Inequalities in System and Control Theory. Society for Industrial and Applied Mathematics (SIAM), 1994.

[29] Bradley Efron and R J Tibshirani. An introduction to the bootstrap. Monographs on Statistics and Applied Probability. Chapman \& Hall / CRC, 1994.

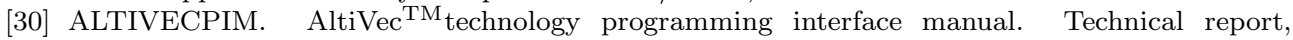
Freescale Semiconductor, Inc., 1999. 